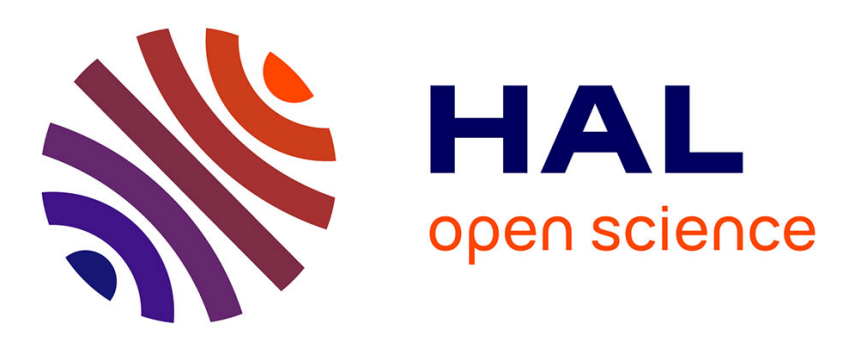

\title{
Mesh Parameterization with Generalized Discrete Conformal Maps
}

Colin Cartade, Christian Mercat, Rémy Malgouyres, Chafik Samir

\section{To cite this version:}

Colin Cartade, Christian Mercat, Rémy Malgouyres, Chafik Samir. Mesh Parameterization with Generalized Discrete Conformal Maps. Journal of Mathematical Imaging and Vision, 2013, 46 (1), pp.1 - 11. 10.1007/s10851-012-0362-y . hal-01598210

\section{HAL Id: hal-01598210 https://hal.science/hal-01598210}

Submitted on 16 Oct 2017

HAL is a multi-disciplinary open access archive for the deposit and dissemination of scientific research documents, whether they are published or not. The documents may come from teaching and research institutions in France or abroad, or from public or private research centers.
L'archive ouverte pluridisciplinaire HAL, est destinée au dépôt et à la diffusion de documents scientifiques de niveau recherche, publiés ou non, émanant des établissements d'enseignement et de recherche français ou étrangers, des laboratoires publics ou privés. 


\title{
Mesh parameterization with generalized discrete conformal maps
}

\author{
Colin Cartade $^{1}$, Christian Mercat ${ }^{2}$, Rémy Malgouyres ${ }^{1}$, and Chafik Samir ${ }^{3}$ \\ 1 Clermont-Université, LIMOS, Complexe des Cézeaux, 63172 Aubière, France \\ \{colin. cartade, remy. malgouyres\}@u-clermont1.fr \\ 2 Université Lyon 1, IUFM, Bât. Braconnier, 69622 Vileurbanne, France \\ christian.mercat@gmail.com \\ 3 Clermont-Université, ISIT, Complexe des Cézeaux, 63172 Aubière, France \\ chafik.samir@u-clermont1.fr
}

\begin{abstract}
We introduce a new method to compute conformal parameterizations using a recent definition of discrete conformity, and establish a discrete version of the Riemann mapping theorem. Our algorithm can parameterize triangular, quadrangular and digital meshes. It can also be adapted to preserve metric properties. To demonstrate the efficiency of our method, many examples are shown in the experiment section.
\end{abstract}

Keywords: conformal parameterization, digital surfaces, Riemann mapping theorem

\section{Introduction}

Parameterizations are one to one maps from 3D discrete surfaces to the Euclidean plane. Parameterizations are widely used in computer graphics allowing one to simplify difficult 3D problems in easy 2D tasks. For instance, texture mapping, a very classical application, boils down to the trivial task of mapping an image on a rectangular domain. Parameterizations also allow to consider a mesh as the image of a function from the plane to the 3D space, and thus work with functions instead of 3D sets. Such a representation is useful for applications as morphing, surface fitting, etc.

A parameterization should preserve the geometrical properties of the mesh: angles (conformal map), areas (authalic maps), lengths (isometric map), etc. It is known that maps which are both conformal and authalic are isometric, and only developable surfaces have an isometric flat parameterization. In practice, one often look for conformal maps. Parameterization preserve angles, lengths ratios locally, and more generally the local aspect of the mesh. It is often sufficient to obtain a good parameterization.

In this paper we present a new algorithm to compute conformal parameterizations using the definition of discrete conformity as given in [14]. Theoretically, it is shown to be a generalization of the cotan conformal coordinates methods [18, $5]$ and it leads to a discrete version of the Riemann mapping theorem with closer boundary conditions than those of classical conformal techniques. In practice, 
the algorithm consists in minimizing a discrete energy measuring conformity. It can be adapted to take care of metric properties such as lengths and areas allowing to compute low-stretch quasi-conformal parameterization with natural boundaries. The energies being expressed in terms of the vertices coordinates and not the angles as ABF method [19], it is also possible to perform constrained texture mapping as shown in Figure 1 (b).

The rest of the paper is organized as follows. In Section 1 we introduce the definition of discrete conformal maps for quadrangular meshes and adaptations for triangular and digital meshes. In Section 2, we discuss boundary conditions ensuring uniqueness. In particular we establish a discrete version of the Riemann mapping theorem and give conditions leading to the same solution as the cotan conformal coordinates methods. In Section 3, we describe the minimization algorithm used to compute parameterization in practice. Various energies are introduced to preserve more or less angles, areas, lengths or the boundary. Numerical illustrations and comparative studies are given in Section 4.

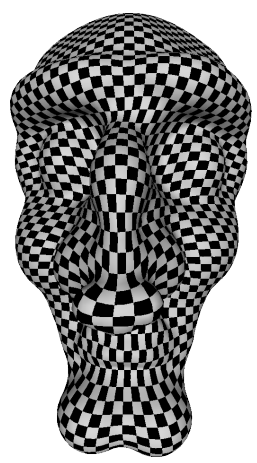

(a)

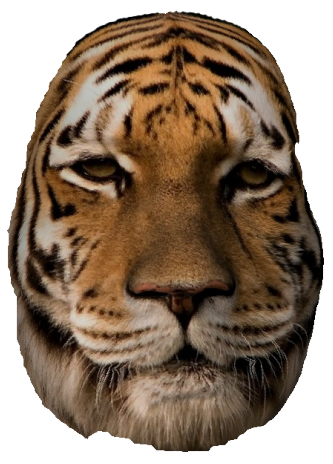

(b)

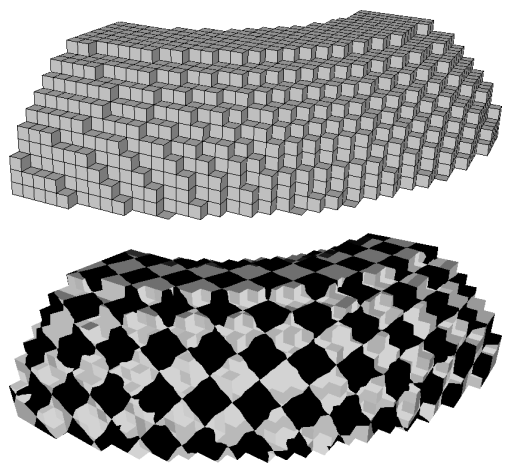

(c)

Fig. 1: Examples of

(a) unconstrained texture mapping of a mesh

(b) constrained texture mapping of a mesh

(c) unconstrained texture mapping of a digital surface

\section{Discrete conformal parameterizations}

\subsection{Case of quadrangular meshes}

In real continuous theory, a surface parameterization is a bijective application from the surface $S$ in $\mathbb{R}^{3}$ to the plane: $(x, y, z) \in S \mapsto(s(x, y, z), t(x, y, z)) \in$ $\mathbb{R}^{2}$. For meshes, it boils down to a point $v^{\prime}=(s, t)$ assigned to each vertex $v=(x, y, z)$. In the sequel, we will identify $v^{\prime}$ with the complex number $s+i t$. 
Locally identifying each face $\left(v_{0}, v_{1}, v_{2}, v_{3}\right)$ of a quad mesh to points in the plane (in one way or another) one can view the diagonals $v_{2}-v_{0}$ and $v_{3}-v_{1}$ as two complex numbers and compute the ratio $\rho=\frac{v_{3}-v_{1}}{i\left(v_{2}-v_{0}\right)}$, which is defined up to a global similarity. Following [14], we call this data a discrete conformal structure and will say that a parameterization is discrete conformal if it preserves the ratios $\rho$. In other words, for all faces of the mesh, we require that

$$
\frac{v_{3}^{\prime}-v_{1}^{\prime}}{v_{2}^{\prime}-v_{0}^{\prime}}=i \rho
$$

Geometrically, such a parameterization preserves the angle between the diagonals and the ratio of their lengths. Intuitively, it corresponds to the following characterization of conformity : a map is conformal if and only if its derivative is a similarity everywhere. For simplicity, we can rewrite (1) as a linear equation

$$
v_{3}^{\prime}-v_{1}^{\prime}=i \rho\left(v_{2}^{\prime}-v_{0}^{\prime}\right),
$$

As a consequence, a conformal parameterization is a solution of a (complex valued) linear system.

Remark 1. Even if the four vertices of a quad are not in the same plane we can define the ratio $\rho$. Indeed, the diagonals in $\mathbb{R}^{3}$, when not colinear, can be viewed as two vectors spanning a plane, wherein the complex ratio can be computed. This choice amounts to defining the normal to the surface as the cross-product of these diagonals. A prior knowledge of the normal, therefore of the tangent plane, is another way to identify the quad-face to a quadrilateral in the complex plane, by projecting the vertices onto this tangent plane. The ratio does not depend on the choice of the normal basis identifying the tangent plane with the complex numbers. Together, all these identifications of the tangent plane at each quad, considered as local charts, form an atlas of the surface.

\subsection{Case of triangular meshes}

In practice, for practical reasons, one often use triangular meshes. The definition can be adapted to this case: we add a new (combinatorial) dual point to each face and to each boundary edge, a standard procedure in remeshing. Then for each edge of the initial mesh we form a quadrangle by joining the extremities of the edge with

1. the two dual points inside the adjacent faces if it is not a boundary edge,

2. the dual points inside the adjacent face and on the edge if it is a boundary edge.

A detailled version of these two steps is given in Figure 2. On the left, we display the initial triangular mesh and the dual points, and on the right the obtained quadrangular faces.

By definition, quads consist of two triangles that do not necessarily belong to the same plane. To determine the $\rho$ coefficient, we rotate one of them until 


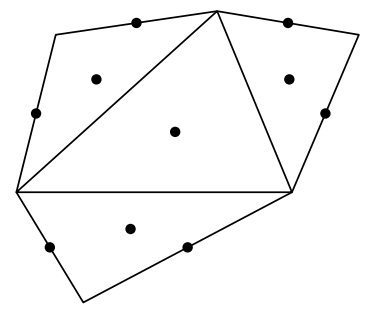

(a)

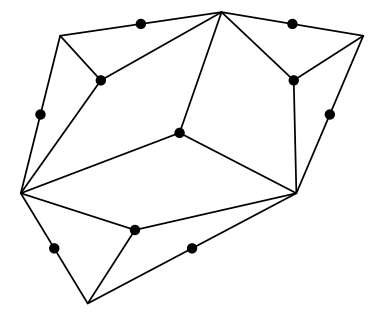

(b)

Fig. 2: Construction of a quadrangular mesh from a triangular one.

One dual point per face and boundary edge, displayed by a small bullet.

(a) Initial triangular mesh and dual points.

(b) Resulting quadrangular mesh, one quad per initial edge.

it belongs to the plane of the second, that is to say we flatten them using the intrinsic metric of the polyhedral surface. Once we have this quad structure and a $\rho$ for each quad we look for a parameterization using the way described in Section 1.1. In particular, we parameterize both the initial vertices and the dual points.

Remark 2. The use of the extrinsic (as in Remark 1) or intrinsic distances does not seem to imply big differences as noted in another context in [2].

\subsection{Case of digital surfaces}

The definition we gave in Section 1.1 needs to be adapted to parameterize digital surfaces whose faces are surfels. Indeed, these faces are planar squares and all the $\rho$ coefficients are equal to 1 . Therefore a more meaningful discrete conformal structure has to be defined, using extrinsic or non local data such as a given normal vector [15]. We compute first a normal vector of each face using the method described in [8]. It allows us to determine the tangent plane of the surface in each surfel. Then, we project the four edgels on this plane, obtaining a parallelogram which better approximates the continuous surface than the initial surfel. Finally, we define the $\rho$ coefficient of a surfel as the one of this projected parallelogram. An example of the construction is depicted in Figure 3.

\section{Boundary conditions and uniqueness}

\subsection{Solutions of the conformal system}

We denote by $n_{f}, n_{e}, n_{b}$ and $n_{v}$ respectively, the number of faces, edges, boundary edges and vertices of the mesh. The linear system (2) consists of $2 n_{f}$ real equations and $2 n_{v}$ real unknowns. As a mesh always has more vertices than faces it does not have a unique solution. 


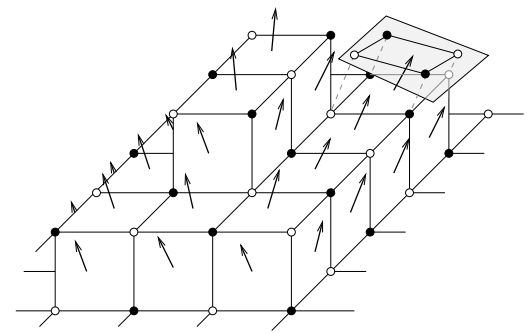

Fig. 3: Definition of the $\rho$ coefficient of a surfel, the one of the parallelogram obtained by projecting the surfel on the tangent plane.

More precisely, the Euler characteristic of the disc is

$$
1=n_{f}-n_{e}+n_{v}
$$

and according to a classical mesh property

$$
4 n_{f}=2 n_{e}-n_{b} .
$$

Adding $2 \times(3)$ to $(4)$ we obtain

$$
2\left(n_{v}-n_{f}\right)=n_{b}+2 .
$$

Hence, in order to ensure uniqueness we need $n_{b}+2$ real constraints.

\subsection{A discrete version of the Riemann mapping theorem}

The Riemann mapping theorem states that each surface which is homeomorphic to the closed unit disc admits a conformal parameterization. Moreover, the holomorphic map is unique if one boundary is mapped to the other one and the images of 3 boundary points are fixed $[1,21,3,9]$.

We can use the $n_{b}+2$ degrees of freedom of the linear system to respect similar boundary conditions. First, we enforce the boundary of the parameterization to remain on the unit circle, adding $n_{b}$ real constraints. Unfortunately, it remains 2 degrees of freedom and we can only fix the images of two boundary vertices. Since discrete conformal maps are known to converge to continuous conformal maps when the faces sizes tends to 0 we would expect similar boundary conditions. So we can wonder where the extra-freedom has gone. The issue is that our system is over-determined. It can be observed by freeding one of the fixed boundary points from begin stuck to the circle. If we only ask it belongs to the tangent line in this point, the solution will eventually be very close to the circle. Therefore fixing three points will give an optimal solution which is of a very low, but in general non null, conformal energy. The process is displayed on Figure 4.

However, our boundary conditions, i.e send the boundary on the circle, fix 2 points and let the third one the tangent line, are much closer to the Riemann theorem than those of other classical discrete conformal algorithms. Indeed, [7, 18] fix all the boundary points and $[5,12]$ fix two boundary points but the other ones are not mapped on the circle. 


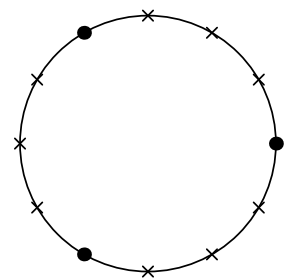

(a)

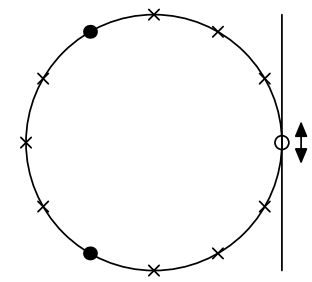

(b)

- point-fixed-on-the-circle

$\times$ point-on-the-circle,-not-fixed

$\circ$ point-on-the-tangent-line,-not-fixed

Fig. 4: (a) Boundary conditions of the Riemann theorem.

(b) Boundary conditions of the discrete version.

\subsection{Generalization of the cotan conformal coordinates methods}

In this section, we will show that discrete conformal parameterization are generalizations of the cotan conformal coordinates methods [18,5]. More precisely, we will show that a convenient choice of the dual points and the additional $n_{b}+2$ boundary constraints lead to the same parameterizations as those computed with these methods.

We remind the reader that they apply to triangular meshes and consist in solving a linear system.

The equations for interior points are the same for both methods: for each interior point $v_{i}$,

$$
\sum_{j: v_{j} \text { neighbour of } v_{i}}\left(\cot \alpha_{i, j}+\cot \beta_{i, j}\right)\left(v_{j}^{\prime}-v_{i}^{\prime}\right)=0
$$

where $\alpha_{i, j}$ and $\beta_{i, j}$ are the opposite angles to the edge $\left[v_{i}, v_{j}\right]$, as show on Figure 5 (a).

They differ for boundary points:

- in [18], the boundary is fixed, often on a convex boundary.

- in $[12,5]$, each boundary coordinate $v_{i}^{\prime}$ verifies

$$
\sum_{\Delta_{i j k}} \cot \alpha_{i, j}\left(v_{i}^{\prime}-v_{j}^{\prime}\right)+\cot \beta_{k, j}\left(v_{i}^{\prime}-v_{k}^{\prime}\right)=i \times \sum_{\Delta_{i j k}}\left(v_{k}^{\prime}-v_{j}^{\prime}\right) .
$$

where the sum is over all the triangles $\Delta_{i j k}=\left(v_{i}, v_{j}, v_{k}\right)$ containing $v_{i}$. Two boundary coordinates have also to be fixed to ensure invariance to translation and rotation. 


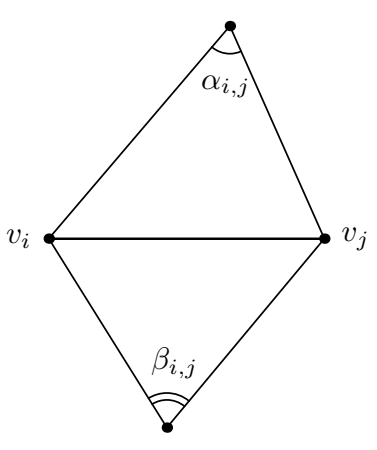

(a)

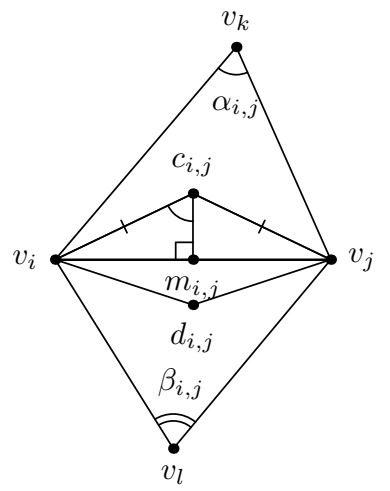

(b)

Fig. 5: (a) Definition of the angles in the cotan formula

(b) Notations used in the proof of Proposition 1

\section{Proposition 1.}

Consider the following choices of dual points and boundary equations

i) the dual points are the circumcenters of the faces and the middles of the boundary edges

ii) the coordinates of the initial (non dual) boundary vertices and one of the dual boundary point are fixed.

iii) the coordinates of two initial boundary points are fixed, the coordinates of dual boundary points are in the middle of the image of boundary initial edges.

With the same choice of fixed points,

1. conditions i) and ii) results in the same coordinates for initial vertices as with the first cotan conformal coordinates method.

2. conditions $i$ ) and iii) results in the same coordinates for initial vertices as with the second cotan conformal coordinates method.

Proof (of 1.).

We remark that ii) consists in $\frac{n_{b}}{2}+1$ complex linear equations, so due to the results of Section 2.1, there exists one paramaterization satisfying i) and ii). We only have to show that the coordinates of initial vertices verify (5).

Consider two adjacent faces of the mesh $\left(v_{i}, v_{j}, v_{k}\right)$ and $\left(v_{i}, v_{l}, v_{j}\right)$. We denote by $c_{i, j}$ and $d_{i, j}$ their circumcenters. An example of the construction is shown on Figure $5(\mathrm{~b})$. We begin by computing the conformal coefficient $\rho_{i, j}$ of the quad $\left(v_{i}, v_{l}, v_{j}, v_{k}\right)$ constructed from the edge $\left[v_{i}, v_{j}\right]$. We also denote by $m_{i, j}$ the middle of $\left[v_{i}, v_{j}\right]$ and by $\alpha_{i, j}$ and $\beta_{i, j}$ the angles in $v_{k}$ and $v_{l}$. 
First, since the angle in $m_{i, j}$ is right, $\rho_{i, j}$ is real:

$$
\rho_{i, j}=\frac{\left\|c_{i, j}-m_{i, j}\right\|}{\left\|v_{j}-v_{i}\right\|}+\frac{\left\|d_{i, j}-m_{i, j}\right\|}{\left\|v_{j}-v_{i}\right\|} .
$$

and

$$
\frac{\left\|c_{i, j}-m_{i, j}\right\|}{\left\|v_{j}-v_{i}\right\|}=\frac{1}{2} \cot v_{i} \widehat{c_{i, j}} v_{j}
$$

Second, due to the inscribed angle theorem

$$
v_{i} \widehat{c_{i, j}} v_{j}=\alpha_{i, j} .
$$

Hence

$$
\rho_{i, j}=\frac{1}{2}\left(\cot \alpha_{i, j}+\cot \beta_{i, j}\right) .
$$

Adding the equations of (2) involving the vertex $v_{i}$ we obtain

$$
\frac{i}{2} \sum_{j}\left(\cot \alpha_{i, j}+\cot \beta_{i, j}\right)\left(v_{j}^{\prime}-v_{i}^{\prime}\right)=i \sum_{j} \rho_{i, j}\left(v_{j}^{\prime}-v_{i}^{\prime}\right)=i \sum_{j}\left(c_{i, j}^{\prime}-d_{i, j}^{\prime}\right)=0,
$$

the last equality being true because the edges $\left[c_{i, j}^{\prime}, d_{i, j}^{\prime}\right]$ form a loop. So $(5)$ is verified.

Proof (of 2.).

Similarly to the proof of 2 ., we only have to prove that the solution verifies (6). For a boundary vertex $v_{i}$, the left hand side of equation (7) becomes

$$
\begin{gathered}
\frac{i}{2}\left(\cot \alpha_{i, i_{1}}\left(v_{i_{1}}^{\prime}-v_{i}^{\prime}\right)+\cot \beta_{i, i_{2}}\left(v_{i_{2}}^{\prime}-v_{i}^{\prime}\right)+\sum_{j: v_{j} \notin \text { boundary }}\left(\cot \alpha_{i, j}+\cot \beta_{i, j}\right)\left(v_{j}^{\prime}-v_{i}^{\prime}\right)\right) \\
=-\frac{i}{2} \sum_{\Delta_{i j k}}\left(\cot \alpha_{i, j}\left(v_{i}^{\prime}-v_{j}^{\prime}\right)+\cot \beta_{k, j}\left(v_{i}^{\prime}-v_{k}^{\prime}\right)\right)
\end{gathered}
$$

where $v_{i_{1}}$ and $v_{i_{2}}$ are the next and previous (initial) boundary points. As for the right hand side, it becomes

$$
\begin{gathered}
c_{i, i_{1}}^{\prime}-m_{i, i_{1}}^{\prime}+m_{i, i_{2}}^{\prime}-d_{i, i_{2}}^{\prime}+\sum\left(c_{i, j}^{\prime}-d_{i, j}^{\prime}\right) \\
=m_{i, i_{2}}^{\prime}-m_{i, i_{1}}^{\prime}=\frac{1}{2}\left(v_{i_{2}}^{\prime}-v_{i_{1}}^{\prime}\right)=\frac{1}{2} \sum_{\Delta_{i j k}}\left(v_{k}^{\prime}-v_{j}^{\prime}\right) .
\end{gathered}
$$

Multiplying (8) and (9) by $2 i$, we obtain (6).

Given this proposition, it appears that the boundary conditions introduced by the Intrinsic parameterization method are no so natural as they can seem at first sight. Moreover our definition of conformity allowing arbitrary dual points can be of interest, when some angles of the triangles are obtuse. Then the circumcenters are not necessarily inside the triangle and the cotan conformal coordinates methods can fail. 


\section{Minimization algorithms}

\subsection{Energy minimization}

Many parameterizations methods, including $[5,7,12,18]$, consist in solving sparse linear systems. As the system of equations (2) is also sparse, we could think of using similar techniques. But the boundary condition, i.e. remaining on a circle, is not linear and even not quadratic. That is why we implement a non-linear minimization technique.

We denote by $\rho_{i, j}$ the $\rho$ coefficient of the face containing the diagonal $\left[v_{i}, v_{j}\right]$. Then we introduce the conformal energy

$$
H=\sum\left|\left(v_{l}^{\prime}-v_{j}^{\prime}\right)-\rho_{i, j}\left(v_{k}^{\prime}-v_{i}^{\prime}\right)\right|^{2}
$$

where the sum is over all the quads $\left(v_{i}, v_{j}, v_{k}, v_{l}\right)$ of the mesh, and the boundary energy

$$
C=\sum\left(\left|v_{i}^{\prime}\right|^{2}-1\right)^{2}
$$

where the sum is over all the boundary vertices $v_{i}$ except the two ones whose parameters are fixed. We search the parameters $v_{i}^{\prime}$ minimizing the total energy

$$
E=\alpha H+\beta C
$$

for chosen positive real coefficients $\alpha$ and $\beta$.

The minimization is performed using the limited-memory BFGS algorithm. It is a quasi-Newton algorithm optimized for large Hessians. Moreover it computes an approximation of the second derivatives of the function from the exact gradient so we only need to compute the first derivative of $E$. Note that the derivative of $H$ can be easily determined using complex numbers. Indeed, if $v_{i}^{\prime}=x_{i}+y_{i}$,

$$
\begin{aligned}
\frac{\partial}{\partial x_{i}}\left|\left(v_{l}^{\prime}-v_{j}^{\prime}\right)-\rho_{i, j}\left(v_{k}^{\prime}-v_{i}^{\prime}\right)\right|^{2} & =2 \operatorname{Re}\left(\bar{\rho}\left(v_{l}^{\prime}-v_{j}^{\prime}-\rho\left(v_{k}^{\prime}-v_{i}^{\prime}\right)\right)\right) \\
\frac{\partial}{\partial y_{i}}\left|\left(v_{l}^{\prime}-v_{j}^{\prime}\right)-\rho_{i, j}\left(v_{k}^{\prime}-v_{i}^{\prime}\right)\right|^{2} & =2 \operatorname{Im}\left(\bar{\rho}\left(v_{l}^{\prime}-v_{j}^{\prime}-\rho\left(v_{k}^{\prime}-v_{i}^{\prime}\right)\right)\right) .
\end{aligned}
$$

We have to take care of initial conditions to reach the right minimum. Indeed, there is no uniqueness in the Riemann theorem when the map is not supposed injective. So the energy $E$ has several (global) minima and with a convenient choice of initial conditions we could perfectly reach a non injective conformal parameterization. Such a parameterization is depicted on Figure 6 (b).

In practice it is efficient to start from an initial parameterization whose interior points are in $(0,0)$ and whose boundary points are on the unit circle with the same distances between them as on the mesh. Then the process unfolds the interior points, more or less like the relaxation of a network of spring. To have less distortion it is generally better to choose the fixed points as far as possible from each other. An example is given in Figure 6 (a) where the fixed points are represented by big dots. 


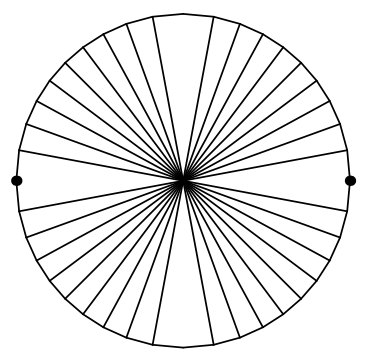

(a)

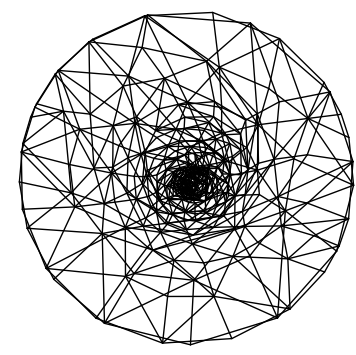

(b)

Fig. 6: (a) Example of initial parameterization, boundary points on the circle, interior points in 0 .

(b) Example of non injective parameterization.

\subsection{Preservation of lengths and areas}

Boundary conditions introduced yet are interesting theoretically due to their connection to the Riemann mapping theorem but not very useful practically since they produce strong length distortions near the boundary. Moreover, even if conformity is the key feature of parameterization, it is not the only criterion to ensure a good texture mapping. Indeed, conformal maps can lead to parameterizations which are very tight in some regions and more sparse in others. If we map a checkerboard with such a parameterization we obtain big squares in the first regions and little ones in the others which is of course unsatisfactory.

To obtain a more natural boundary and reduce metric distortions we propose to replace the energy term $C$ by other ones measuring metric distortions. Precisely, we introduce three new cost functions $L^{\prime}, L$ and $A$ attached to preserve respectively lengths of boundary edges, lengths of all edges, and areas of faces

$$
\begin{aligned}
& L=\sum_{\substack{\text { boundary edge } \\
\left[v_{i}, v_{j}\right]}}\left(\left|v_{i}^{\prime}-v_{j}^{\prime}\right|^{2}-\left\|v_{i}-v_{j}\right\|^{2}\right)^{2}, \\
& L=\sum_{\text {edge }\left[v_{i}, v_{j}\right]}\left(\left|v_{i}^{\prime}-v_{j}^{\prime}\right|^{2}-\left\|v_{i}-v_{j}\right\|^{2}\right)^{2}, \\
& A=\sum_{\substack{\text { face } \\
\left(v_{i}, v_{j}, v_{k}, v_{l}\right)}}\left(\left(\operatorname{Im}\left(v_{j}^{\prime}-v_{i}^{\prime}\right) \overline{\left(v_{k}^{\prime}-v_{i}^{\prime}\right)}-\left\|\left(v_{j}-v_{i}\right) \wedge\left(v_{k}-v_{i}\right)\right\|\right)^{2}\right. \\
& \left.+\left(\operatorname{Im}\left(v_{k}^{\prime}-v_{i}^{\prime}\right) \overline{\left(v_{l}^{\prime}-v_{i}^{\prime}\right)}-\left\|\left(v_{k}-v_{i}\right) \wedge\left(v_{l}-v_{i}\right)\right\|\right)^{2}\right)
\end{aligned}
$$

and we propose to minimize the energy

$$
E=\alpha H+\beta A+\gamma L+\delta L^{\prime}
$$

where $\alpha, \beta, \gamma$ and $\delta$ are nonnegative real numbers to be chosen. 
As any isometric transformation of a given parameterization has the same energy we have to fix the image of one boundary point and the slope of the next boundary edge to ensure uniqueness of the solution. For numerical reasons the algorithm described in previous section does not converge in general towards the right local minimum if we use the same initial conditions. Instead we propose the following two steps minimization.

1. minimize $H$ with a fixed boundary

2. use this minimum as initial condition to minimize $E$.

Step 1 being much faster with respect to step 2, it does not affect convergence speed to perform two minimizations. Moreover, at step 1 we can relax the stopping criteria since we need not to converge the exact minimum. We only want to unfold a little the parameterization to be closer to the minimum and allow step 2 to converge.

Remark 3. Another classical Riemann-Hilbert condition [21] is to preserve boundary metric. The use of energy $E=H+L^{\prime}$ allows to be close to this condition. It can also be useful in practice since it is faster to compute $L^{\prime}$ than $L$ or $A$.

Remark 4. For a given quad $\left(v_{0}, v_{1}, v_{2}, v_{3}\right)$ our area energy preserve independently the areas of two triangles $\left(v_{0}, v_{1}, v_{2}\right)$ and $\left(v_{0}, v_{2}, v_{3}\right)$ instead of the area of the whole quad directly. It avoids the formation of non convex quads when the area coefficient $\beta$ is high.

\subsection{Stabilizing the boundary}

When using the boundary metric energy $L^{\prime}$, for numerical reason, little artefacts can appear along the boundary. An example of boundary with two such artefacts is shown on Figure 7. This problem can also occur, but less frequently, using energies $L$ and $A$ with very low coefficient $\alpha$ and $\beta$. In this section we present improvments to get a greater numerical precison for boundary vertices.

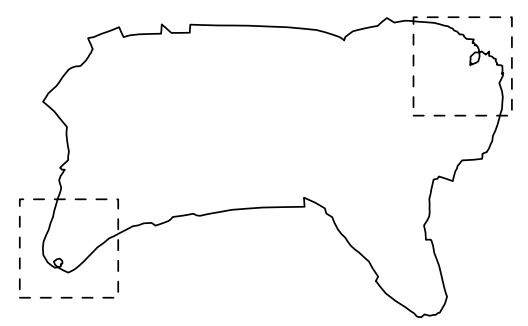

(a)

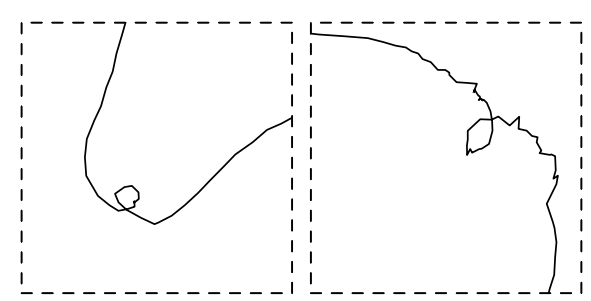

(b)

Fig. 7: Little artefacts on the boundary using the boundary metric energy $L^{\prime}$ (a) Whole boundary (b) Zoom in on critical regions 
The sum of the exterior angles of any polygon is $2 \pi$. And each little artefact of Figure 7 increase this sum of $2 \pi$. So if we could preserve the boundary angles we would prevent the formation of these artefacts. Thus we propose to add an energy term to preserve angles between boundary edges. To have a differentiable energy, in the same way as conformity, we introduce complex coefficients measuring both angles and lengths ratios. More precisely, for each boundary vertex $v_{i}$, we denote by $p(i)$ and $n(i)$ the indices of the previous and next vertex along the boundary and associate to $v_{i}$ the complex number $\rho=r e^{i \theta}$ where

$$
r=\frac{\left\|v_{n(i)}-v_{i}\right\|}{\left\|v_{p(i)}-v_{i}\right\|}
$$

and the argument $\theta$ is the sum of the angles in $v_{i}$. In case of Figure 8,

$$
\theta=\alpha_{1}+\alpha_{2}+\alpha_{3}
$$

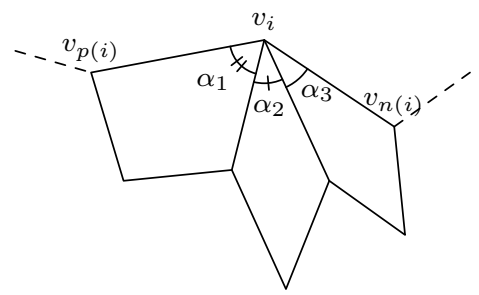

Fig. 8: An example of definition of $\rho=r e^{i \theta}$ for the boundary vertex $v_{i}$,

$$
\rho=r e^{i \theta} \text { with } r=\frac{\left\|v_{n(i)}-v_{i}\right\|}{\left\|v_{p(i)}-v_{i}\right\|} \text { and } \theta=\alpha_{1}+\alpha_{2}+\alpha_{3} \text {. }
$$

Then we introduce the cost function $B$

$$
B=\sum\left|z_{n(i)}^{\prime}-z_{i}^{\prime}-\rho_{i}\left(z_{i}^{\prime}-z_{p(i)}^{\prime}\right)\right|^{2} .
$$

Remark 5. As the conformal energy $H$ already intends to preserve boundary angles, energy $B$ is a little redundant. But it is important numerically: in a way we strengthen the conformal energy for boundary vertices to force the boundary to remain valid all along the process.

Remark 6. The definition of $\rho$ described in this section can also be used to compute parameterizations of a triangular meshes without constructing a quadrangular ones. Indeed we can define for each triangle $\left(v_{0}, v 1, v 2\right)$ the coefficient $\rho$ as $\frac{v_{2}-v_{0}}{v_{1}-v_{0}}$, to preserve the angle and the length ratio between the edges $\left[v_{0}, v_{1}\right]$ and $\left[v_{0}, v_{2}\right]$ and thus the shape of the triangle. All the previously detailed methods can be adapted to this definition. It has the advantage of being simpler and needing less computation time. However the definition depends on the choice of $v_{0}$, and the results of Section 2 are no longer valid. 


\section{Numerical results}

\subsection{Unconstrained parameterization of mesh}

In this section parameterizations of triangular meshes are computed with different coefficients $\alpha, \beta$, etc, to study the influences of the different energies. We also give comparisons with ABF method.

Remind that the energies do not share the same dimension: $H$ has the dimension of an area, $L$ and $L^{\prime}$ of a length to the power 4 and $A$ of an area to the

power 2. We first normalize them in order to have coefficients more independent of the mesh. We denote by $a, l$ and $l^{\prime}$ respectively the mean of the faces areas, the edges lengths and the boundary edges lengths. And we divide $H$ and $B$ by $a, A$ by $a^{2}, L$ by $l^{4}$ and $L^{\prime}$ by $l^{\prime 4}$.

Texture mappings are shown on Figure 9 and statistical features displayed in Table 1. The line "angles" give the mean angular error (not the the value of $H$ ). To measure area distortions, we compute for each face the ratio $\sqrt{\min \left(\frac{a_{p}}{a_{m}}, \frac{a_{m}}{a_{p}}\right)}$ where $a_{m}$ is the area of the face and $a_{p}$ the area of the corresponding face in the parameterization. It should be close to 1 . The line "areas" give the mean of these ratios. The line "lengths" give the mean ratio of edges lengths in the same way.

We observe in practice that the use of an average of $H$ and $L^{\prime}$ allows to compute conformal paramaterizations with a natural boundary but a little more stretch than $\mathrm{ABF}$. Using energy $E=\alpha H+\beta A$ with a small area coefficient $\beta$ also leads to conformal parameterization and reduce the stretch. In particular, the choice $\alpha=100$ and $\beta=1$ gives for most meshes results very similar to ABF. An advantage of the method, is that we can also relax the conformal equation and use a high area coefficient to better preserve areas. Energy $E=H+10 A$ seems to be a good choice to obtain a visually authalic texture mapping. As for energy $L$, it is less useful since it tends to produce folds when we increase its coefficient.

Our method also proves to be very efficient with unnatural boundaries as shown on Figure 10 and 9.

Remark 7. We did not show the texture mapping of ABF since it was almost the same as with $E=100 H+A$. 

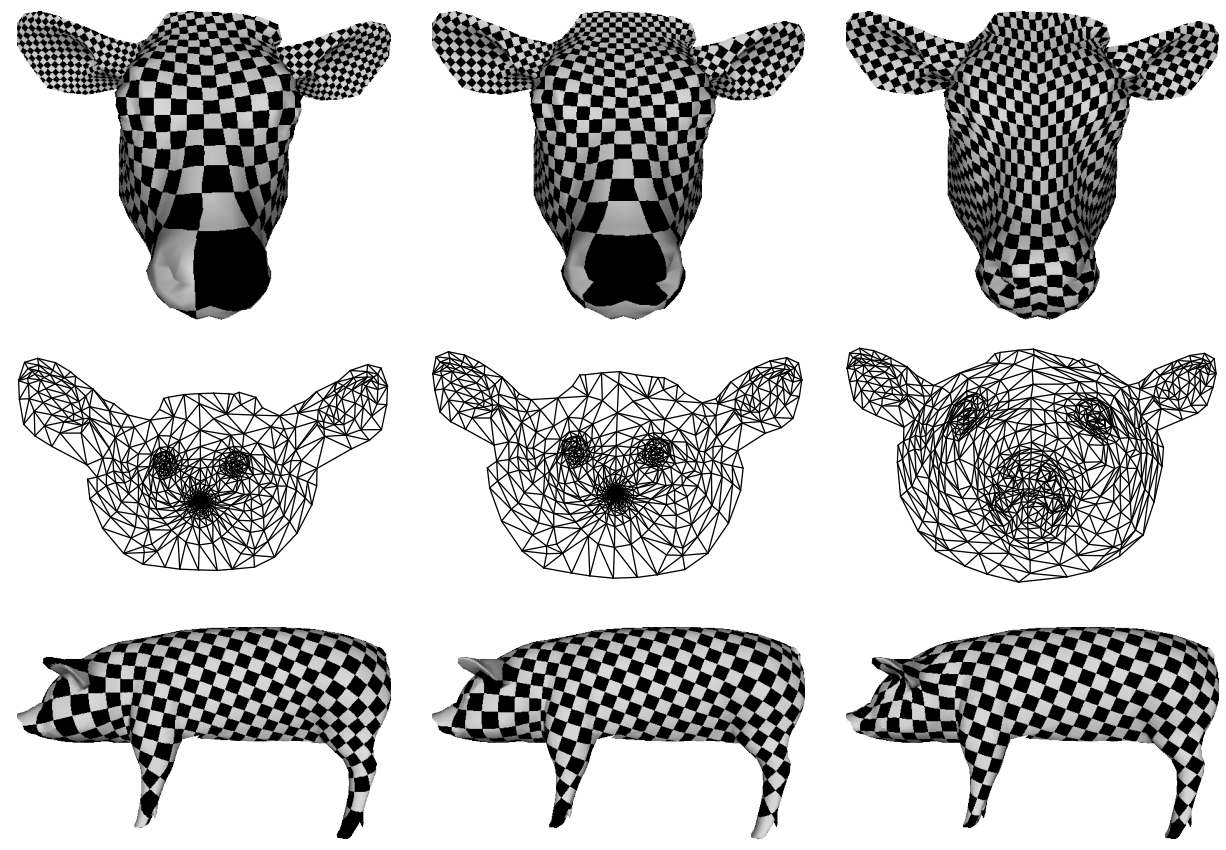

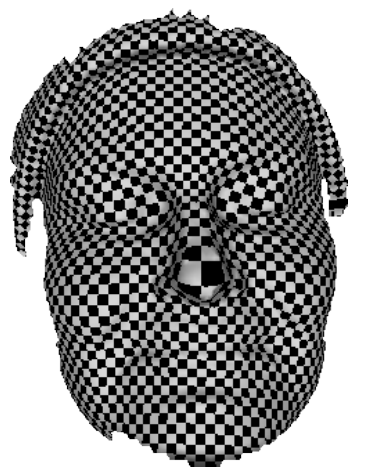

(a)

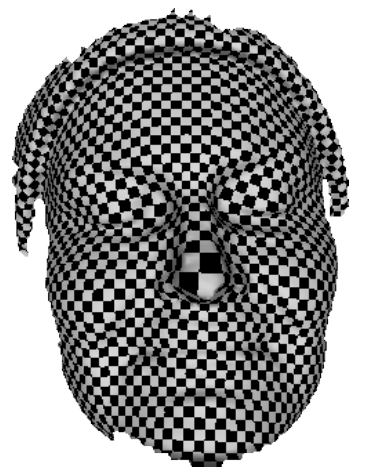

(b)

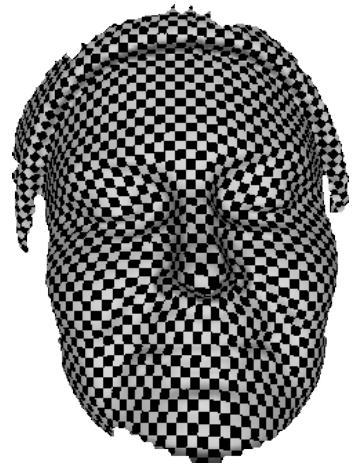

(c)

Fig. 9: Examples of texture mappings with different cost functions
(a) $E=H+L^{\prime}$, conformal and isometric along the boundary
(b) $E=100 H+A$, conformal, more area preserving, similar to $\mathrm{ABF}$
(c) $E=H+10 A$, quasi-conformal, area preserving. 


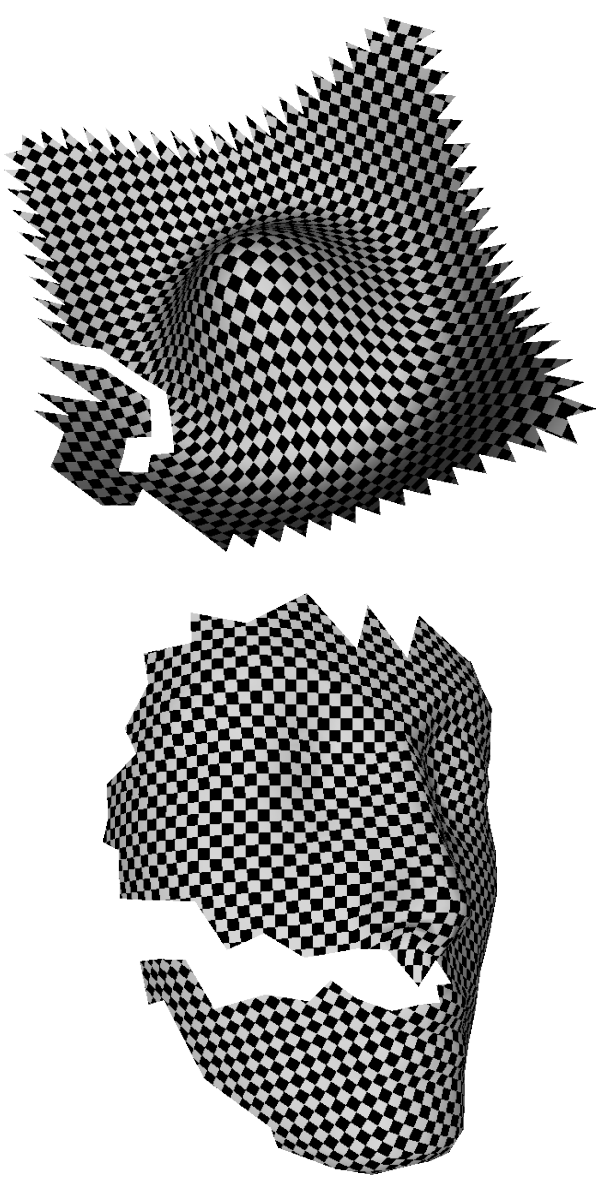

(a)
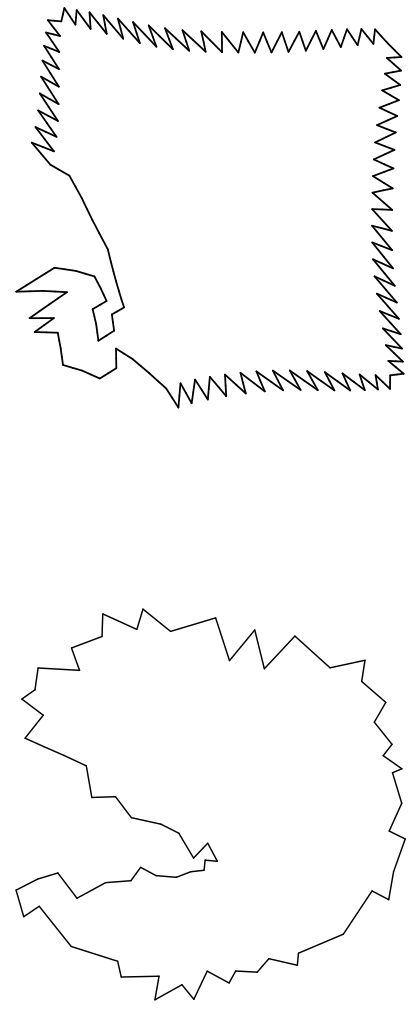

(b)

Fig. 10: Examples of parameterization of meshes with unatural boundaries

(a) Texture mapping

(b) Boundary of the parameterization 


\begin{tabular}{|l|c|c|c|c|}
\hline cow (2,000 faces) & $E=H+L^{\prime}+B$ & $E=100 H+A$ & $E=H+10 A$ & $\mathrm{ABF}$ \\
\hline time (sec) & 1.32 & 0.94 & 0.87 & \\
angles (rad) & 0.06 & 0.06 & 0.25 & 0.05 \\
areas & 0.48 & 0.62 & 0.85 & 0.63 \\
lengths & 0.41 & 0.56 & 0.81 & 0.58 \\
\hline
\end{tabular}

\begin{tabular}{l|l|l|l|l|}
\hline $\operatorname{pig}(6,000$ faces $)$ & $E=H+L^{\prime}+B$ & $E=100 H+A$ & $E=H+10 A$ & $\mathrm{ABF}$
\end{tabular}

\begin{tabular}{|l|c|c|c|c|}
\hline time $(\mathrm{sec})$ & 8 & 11 & 15 & \\
angles $(\mathrm{rad})$ & 0.07 & 0.06 & 0.87 & 0.05 \\
areas & 0.58 & 0.71 & 0.20 & 0.68 \\
lengths & 0.51 & 0.66 & 0.79 & 0.62 \\
\hline
\end{tabular}

face $\left(30,000\right.$ faces $E=H+L^{\prime}+B|E=100 H+A| E=H+10 A$ ABF

\begin{tabular}{|l|c|c|c|c|}
\hline time (sec) & 50 & 33 & 36 & \\
angles (rad) & 0.01 & 0.01 & 0.07 & 0.01 \\
areas & 0.93 & 0.93 & 0.98 & 0.93 \\
lengths & 0.93 & 0.94 & 0.98 & 0.93 \\
\hline
\end{tabular}

Table 1: Quantitative comparison of parameterizations

\subsection{Digital surfaces}

We also show parameterizations of digitals surfaces. Normal vectors are computed using the convolution method of [8]. We observe that remarks of previous section concerning the choice of energies and coefficients apply to the digital case too. Two examples are displayed on Figure 11.

It is interesting to compare our digital method with non voxels ones such as ABF. On the one hand we obtain better texture mappings. Indeed, as shown on Figure 12, due to the use of smoother normals, the checkerboard squares are smoother than those of ABF. On the other hand, when on look at a (plane) conformal parameterization we often have an impression of relief. It is also the case with our digital parameterization since we clearly distinguish little cubes. It is much less clear with the ABF parameterization. 


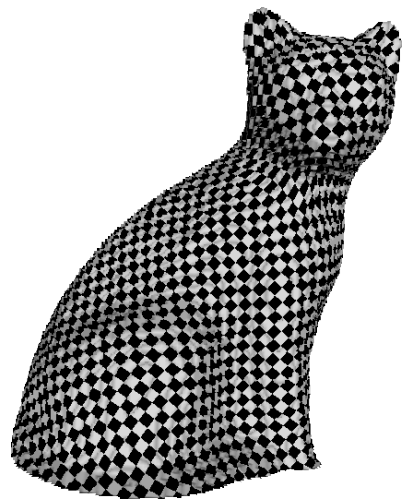

(a)

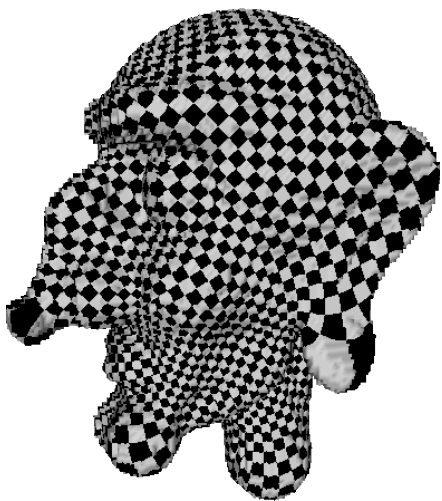

(b)

Fig. 11: Examples of texture mapping on digital surfaces

(a) Cat quasi-conformal parameterization with $E=H+10 A$.

(b) Half-head conformal parameterization with $E=100 H+A$
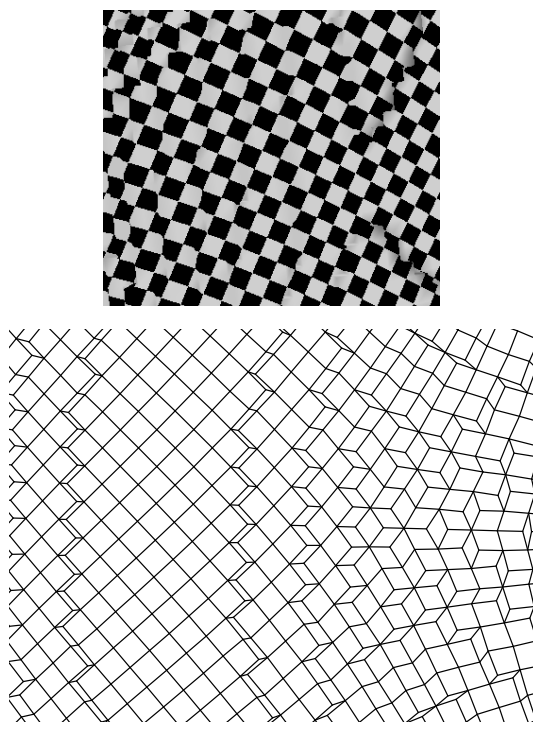

(a)
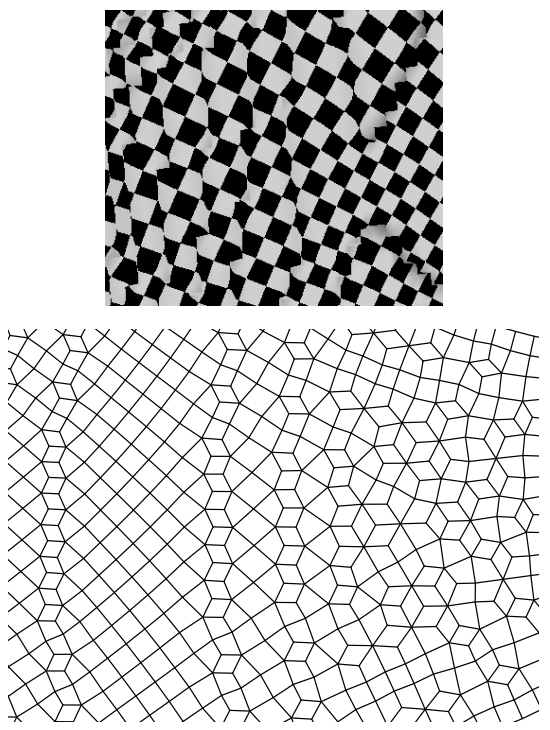

(b)

Fig. 12: Comparison of digital and ABF methods. Zoom in on the parameterization of the cat (Figure $11(\mathrm{a})$ ).
(a) Digital method
(b) ABF method. 


\subsection{Constrained texture mapping}

Another important application of parameterization techniques is texture mapping of a $2 \mathrm{D}$ image on a $3 \mathrm{D}$ model. The main features of the image and the model must fit. Therefore positions of the corresponding points of the parameterization must be fixed. Our method can be adapted to that case. In fact it only reduces the number of variables of the function to minimize: we consider the points that are not fixed.

In the example of Figure 14 we map an image of a face on a mask of Nefertiti. We select manually 13 corresponding points. They are displayed with thin points on the figure. On Figure 14 (a), we map the image on the mesh to see that the 13 points are mapped to the right position. On Figure 14 (b) we display the map of a checkerboard with the same parameterization, it shows that the parameterization is still conformal.
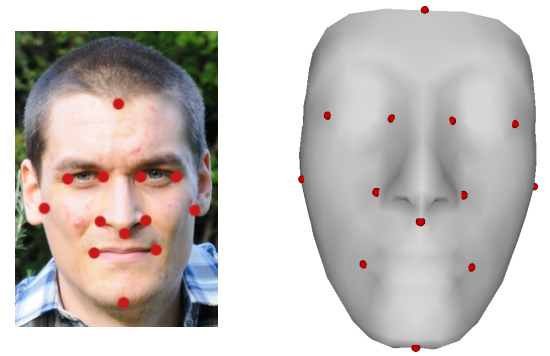

Fig. 13: Corresponding 13 points in the image and the mesh.

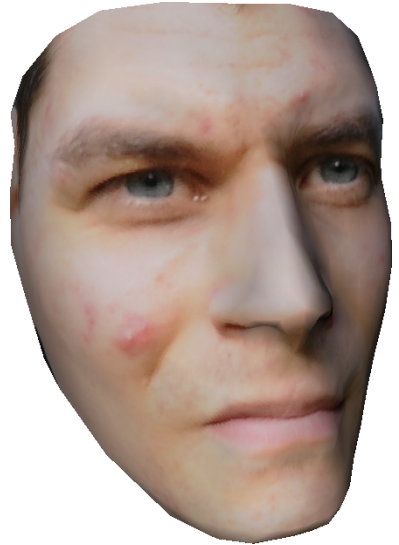

(a)

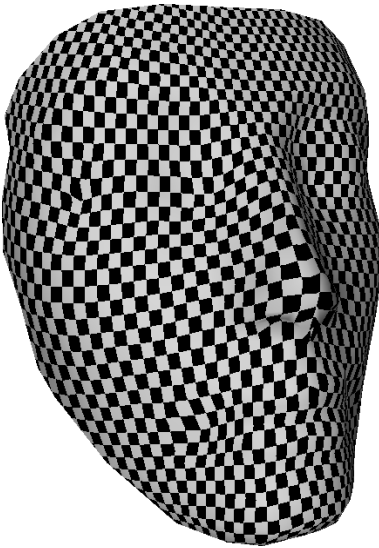

(b)

Fig. 14: Textured 3D model with (a) an image face and (b) a checkerboard. Same parameterization computed with $E=H+0.01 A$. 


\section{Conclusion}

We have described a new method of conformal parameterization that can be applied to different meshes, including quadrangular meshes and digital surfaces. An important feature of our approach is the use of a recent definition of discrete conformity that permits to have discrete theorems close to the continuous ones. Moreover, it is very flexible since the use of different cost functions allows to preserve more or less shapes, the metric, the boundary or positions. Many experimental results are shown to illustrate the different possibilities.

Acknowledgement. This work was partially supported by the ANR project KIDICO (ANR-2010-BLAN-0205-02).

\section{References}

1. Ahlfors, L.V.: Complex analysis. McGraw-Hill Book Co., New York, third edn. (1978), an introduction to the theory of analytic functions of one complex variable, International Series in Pure and Applied Mathematics

2. Bobenko, A.I., Mercat, C., Schmies, M.: Computational Approach to Riemann Surfaces, chap. Conformal Structures and Period Matrices of Polyhedral Surfaces. Springer-Verlag (2011)

3. Bobenko, A.I., Schröder, P., Sullivan, J.M., Ziegler, G.M. (eds.): Discrete differential geometry, Oberwolfach Seminars, vol. 38. Birkhäuser Verlag, Basel (2008), http://dx.doi.org/10.1007/978-3-7643-8621-4, papers from the seminar held in Oberwolfach, May 30-June 5, 2004

4. Clarenz, U., Litke, N., Rumpf, M.: Axioms and variational problems in surface parameterization. Computer Aided Geometric Design 21(8), 727-749 (2004)

5. Desbrun, M., Meyer, M., Alliez, P.: Intrinsic parameterizations of surface meshes. In: Computer Graphics Forum. vol. 21, pp. 209-218 (2002)

6. Esbelin, H., Malgouyres, R.: Convergence of binomial-based derivative estimation for $C^{2}$ noisy discretized curves. In: Discrete Geometry for Computer Imagery. pp. 57-66. Springer (2009)

7. Floater, M.: Mean value coordinates. Computer Aided Geometric Design 20(1), 19-27 (2003)

8. Fourey, S., Malgouyres, R.: Normals estimation for digital surfaces based on convolutions. Computers \& Graphics 33(1), 2-10 (2009)

9. Gu, X.D., Yau, S.T.: Computational conformal geometry, Advanced Lectures in Mathematics (ALM), vol. 3. International Press, Somerville, MA (2008), with 1 CD-ROM (Windows, Macintosh and Linux)

10. Kharevych, L., Springborn, B., Schröder, P.: Discrete conformal mappings via circle patterns. ACM Transactions on Graphics (TOG) 25(2), 438 (2006)

11. Lenoir, A.: Fast estimation of mean curvature on the surface of a 3 d discrete object. In: DGCI '97: Proceedings of the 7th International Workshop on Discrete Geometry for Computer Imagery. pp. 175-186. Springer-Verlag, London, UK (1997)

12. Lévy, B., Petitjean, S., Ray, N., Maillot, J.: Least squares conformal maps for automatic texture atlas generation. ACM Transactions on Graphics 21(3), 362$371(2002)$ 
13. Liu, D., Nocedal, J.: On the limited memory bfgs method for large scale optimization. Mathematical programming 45(1), 503-528 (1989)

14. Mercat, C.: Discrete Riemann surfaces and the Ising model. Communications in Mathematical Physics 218(1), 177-216 (2001)

15. Mercat, C.: Discrete complex structure on surfel surfaces. In: Discrete Geometry for Computer Imagery. pp. 153-164. Springer (2008)

16. Mullen, P., Tong, Y., Alliez, P., Desbrun, M.: Spectral conformal parameterization. In: Computer Graphics Forum. vol. 27, pp. 1487-1494. Wiley Online Library (2008)

17. Nocedal, J.: Updating quasi-newton matrices with limited storage. Mathematics of computation 35(151), 773-782 (1980)

18. Pinkall, U., Polthier, K.: Computing discrete minimal surfaces and their conjugates. Experimental mathematics 2(1), 15-36 (1993)

19. Sheffer, A., de Sturler, E.: Parameterization of faceted surfaces for meshing using angle-based flattening. Engineering with Computers 17(3), 326-337 (2001)

20. Tutte, W.: Convex representations of graphs. Proceedings of the London Mathematical Society 3(1), 304 (1960)

21. Wegert, E.: Nonlinear Riemann-Hilbert problems - history and perspectives. In: Computational methods and function theory 1997 (Nicosia), Ser. Approx. Decompos., vol. 11, pp. 583-615. World Sci. Publ., River Edge, NJ (1999) 